\title{
Is rotating between static and dynamic work beneficial for our fatigue state?
}

\author{
Tessy Luger ${ }^{\mathrm{a}, \mathrm{b}, \mathrm{c}, *}$, Tim Bosch ${ }^{\mathrm{b}, \mathrm{c}}$, Marco J.M. Hoozemans ${ }^{\mathrm{a}}$, DirkJan H.E.J. Veeger ${ }^{\mathrm{a}, \mathrm{d}}$, Michiel P. de Looze $^{\mathrm{a}, \mathrm{b}, \mathrm{c}}$ \\ ${ }^{a}$ Department of Human Movement Sciences, Faculty of Behavioural and Movement Sciences, Vrije Universiteit Amsterdam, MOVE Research Institute Amsterdam, Van \\ der Boechorststraat 9, NL-1081 TB Amsterdam, The Netherlands \\ b TNO, Postbus 3005, NL-2301 DA Leiden, The Netherlands \\ c Body@Work, Research Centre of Physical Activity, Work and Health, TNO-VU/VUmc, Van der Boechorststraat 9, NL-1081 TB Amsterdam, The Netherlands \\ ${ }^{\mathrm{d}}$ Section BioMechatronics \& BioRobotics, Department of BioMechanical Engineering, Faculty of Mechanical, Maritime and Materials Engineering, Delft University of \\ Technology, Postbus 5, NL-2600 AA Delft, The Netherlands
}

\section{A R T I C L E I N F O}

\section{Article history:}

Received 9 December 2015

Received in revised form 29 March 2016

Accepted 5 April 2016

\section{Keywords:}

Task rotation

Perceived fatigue

Manifestations of muscle fatigue

Shoulder

Multichannel electromyography

\begin{abstract}
A B S T R A C T
Shoulder disorders comprise a large part of work-related musculoskeletal disorders. Risk factors, such as repetitiveness and monotony, may cause muscle fatigue and be attenuated by task rotation. We investigated rotation between a dynamic box-lifting task and a relatively static pick-and-place task and aimed to determine whether (1) a high rotation frequency leads to less fatigue development than a low rotation frequency, and (2) a self-selected rotation frequency leads to less fatigue development than imposed rotation frequencies. Ten participants performed four one-hour rotation schedules: two low frequency rotation schedules rotating at $30 \mathrm{~min}$, one high frequency rotation schedule rotating every sixth minute, and a self-selected rotation schedule. Borg, SOFI and electromyography of Trapezius and Deltoid subparts served as fatigue indicators. We found significant signs of fatigue for most schedules regarding the Borg and SOFI ratings and the M. Trapezius pars Descendens. Task rotation frequency had no significant effect on any of the outcome parameters, whereas the self-selected rotation schedule clearly resulted in less development of perceived fatigue than imposed schedules. In conclusion, we think that freedom of rotation has the greatest potential to attenuate potential development of musculoskeletal disorders and we require due caution with the use and interpretation of EMG indicators of fatigue.
\end{abstract}

(c) 2016 Elsevier Ltd. All rights reserved.

\section{Introduction}

In industrialised countries, shoulder disorders comprise a large part of work-related musculoskeletal disorders (Linaker and Walker-Bone, 2015). Important risk factors for the development of shoulder disorders are repetitiveness and monotony of work tasks (Bernard, 1997; Larsson et al., 2007). These factors result in prolonged muscle loading and may cause manifestations of shoulder muscle fatigue (Rashedi and Nussbaum, 2015), an important precursor of musculoskeletal disorders (Straker and Mathiassen, 2009). Task rotation may create opportunities for the muscle to recover from the continuous workload, and, as such, may be an effective strategy to reduce the total exposure to repetitive or

\footnotetext{
* Corresponding author at: Department of Human Movement Sciences, Faculty of Behavioural and Movement Sciences, Vrije Universiteit Amsterdam, MOVE Research Institute Amsterdam, Van der Boechorststraat 9, NL-1081 TB Amsterdam, The Netherlands.

E-mail address: t.luger@vu.nl (T. Luger).
}

monotonous work and reduce the development of muscle fatigue (Howarth et al., 2009; Jonsson, 1988; Mathiassen, 2006).

Recent studies showed that task rotation has no consistent effects on physiological and perceptual outcome parameters. A few studies showed that rotating between refuse collecting, street sweeping and truck driving within or between days can decrease ratings of discomfort and exertion (Kuijer et al., 1999, 2004) and decrease the need for recovery (Kuijer et al., 2005). These studies rotated between tasks that differed considerably in the type of task and also the muscles activated during each task. Two studies that specifically rotated between tasks, which only differed in the load intensity during static shoulder exertions (Horton et al., 2012) and lifting (Horton et al., 2015), did not find changes in manifestations of muscle fatigue. Raina and Dickerson (2009) investigated the alternation between two fairly similar tasks, shoulder flexion and shoulder abduction, which have a different muscular demand; however, they did not find significant effects of rotation on manifestations of muscle fatigue or perceived exertion either. As can be noted from these findings, most research has focussed on the 
implementation of task rotation and its effects on physical demands whereas only the Horton et al. studies $(2012,2015)$ also investigated rotation frequency. In this respect, we would like to assess the rotation effect between two assembly-like tasks which predominantly activate the shoulder-neck muscles but do not differ too much in the load intensity.

Hand-arm tasks, which particularly load the shoulder-neck muscles, can either be relatively static (e.g., Raina and Dickerson, 2009) or more dynamic (e.g., Horton et al., 2015). Several studies investigated differences in physical demands between static and dynamic arm work at similar force levels. Christensen et al. (1995) found no significant differences in EMG between both work types, which, at lower intensities, may be due to very similar recruitment patterns (Søgaard, 1995). In contrast, other studies found that dynamic arm work was perceived less painful and strenuous (Frey Law et al., 2010) and resulted in lower blood pressure values (Genaidy et al., 1990) than static arm work at similar endurance times and force levels, respectively. In general, rotating between static and dynamic hand-arm tasks might result in changed motor unit recruitment and derecruitment (e.g., Madeleine, 2010; Søgaard, 1995) and allow greater blood perfusion to muscles (Frey Law et al., 2010), thus reducing the development of both perceived and muscular fatigue.

This study aimed to determine the influence of task rotation frequency and autonomy on perceived fatigue and manifestations of muscle fatigue during one-hour rotation schedules between a static and dynamic task with an equal overall intensity. We hypothesised that a higher task rotation frequency would increase the opportunities for muscles to recover from the repetitive loading, resulting in less manifestations of perceived and muscle fatigue.

\section{Methods}

\subsection{Participants}

Ten healthy right-handed subjects ( $5 \mathrm{M}, 5 \mathrm{~F}$ ) participated in this study (mean age 26.0 years [SD 3.1], mean weight $65.5 \mathrm{~kg}$ [SD 7.3], mean height $176.2 \mathrm{~cm}$ [SD 8.7]). None of the subjects had prior experience in manual materials handling. They did not report any history of musculoskeletal disorders at the upper extremity and did not perform any heavy exercise $24 \mathrm{~h}$ prior to the experiment. After the participants were informed about the objectives of the experiment, they signed an informed consent. The Ethical Committee of the Faculty of Human Movement Sciences in Amsterdam approved the protocol.

\subsection{Experimental tasks}

In each rotation schedule, participants rotated between a static and a dynamic task (Fig. 1). The cycle time of both tasks was $60 \mathrm{~s}$, their duty cycle $55 \mathrm{~s}$ with a 5-s recovery within each work cycle. For the static pick-and-place task participants bimanually filled and emptied 30 holes with pins (weight $1.3 \mathrm{~g}$ ) using a Pegboard (Purdue Pegboard Model 32020; Lafayette Instrument Company, Lafayette, IN, USA; Fig. 2). For the dynamic box-lifting task participants lifted a box according to a certain trajectory for 12 times within one cycle (weight $1.0 \mathrm{~kg}, L \times W \times H=30 \times 20 \times 25 \mathrm{~cm}$; Fig. 2).

The experimental set-up was anthropometrically adjusted to the subject: the sitting height of the stool was adjusted to a $90^{\circ}$ knee angle; the average working heights for the pick-and-place

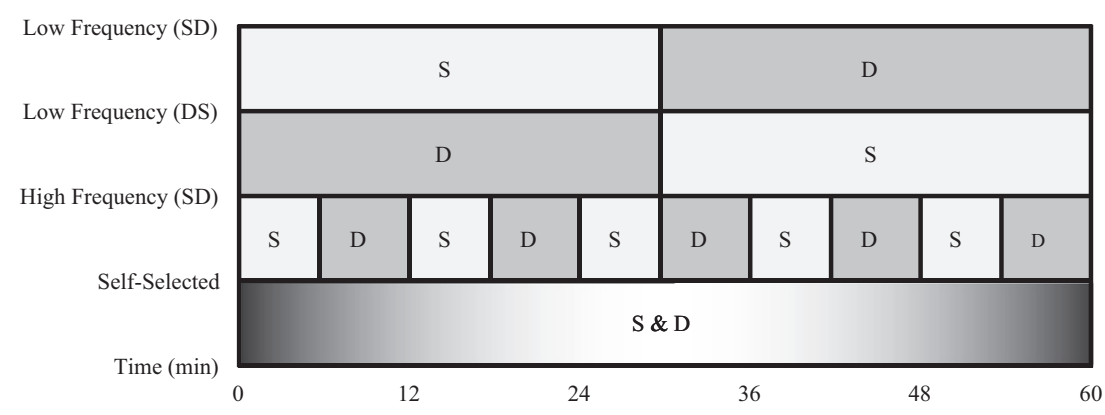

Fig. 1. Schematic representation of the four experimental rotation schedules. $S=$ static; $D=$ dynamic.
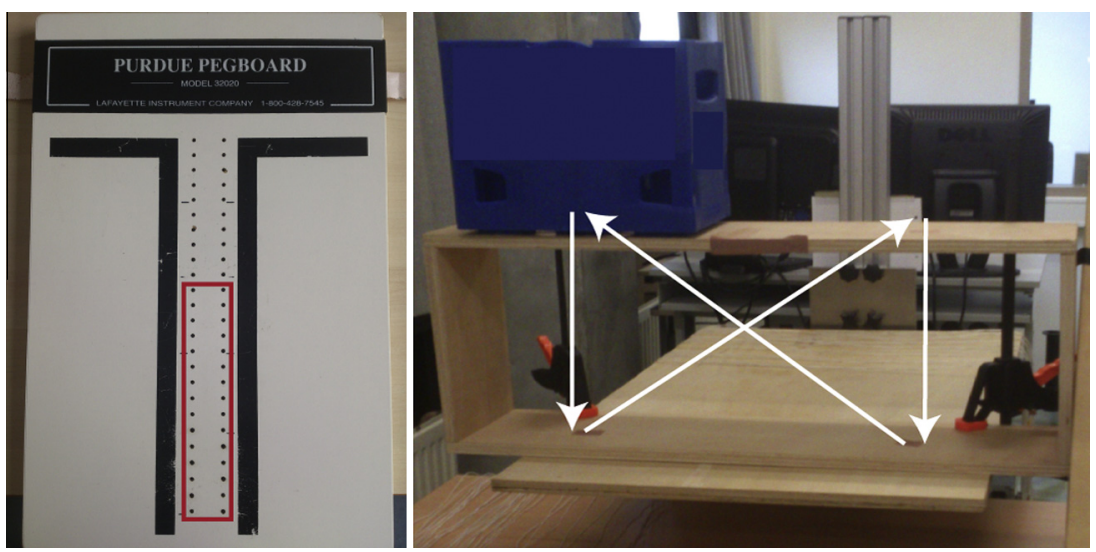

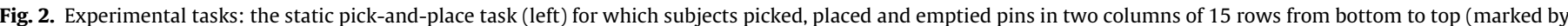

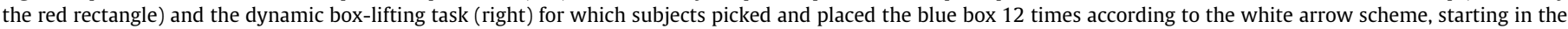
left-up corner. (For interpretation of the references to colour in this figure legend, the reader is referred to the web version of this article.) 
task (performed while seating) and the box lifting task (performed while standing) were adjusted to an average upper arm elevation angle of $30^{\circ}$ relative to the thorax (Wu et al., 2005).

\subsection{Procedure}

The participants performed four one-hour rotation schedules equally spread over two days, where each pair of schedules had a 30 -min rest break in between. Three rotation schedules had a predetermined rotation frequency during which subjects rotated one time starting with either static (low frequency SD) or dynamic (low frequency DS) or nine times starting with static (high frequency SD). The fourth self-selected rotation schedule included 30 min of both tasks and had to be performed in a self-selected order. Overall production was equal in all rotation schedules. The order of the three standardised rotation schedules was systematically varied over the subjects; the self-selected rotation schedule was always performed last so that all participants would have a similar reference frame. At the first day, training sessions for the two tasks were performed until the subject was able to perform the tasks at the required pace. During the entire experiment, subjects were instructed to perform both tasks as fluently as possible according to the timer placed in front of them. The experimenter verbally encouraged and corrected the participants when necessary.

\subsection{Measurements}

\subsubsection{Rating of perceived fatigue}

Before the start, every 6 min during and directly after each rotation schedule, the participants rated perceived fatigue (RPF) in their right shoulder using the CR10 Borg scale (Borg, 1982). Perceived fatigue was also determined before and after each rotation schedule using the Swedish Occupational Fatigue Inventory (SOFI; Åhsberg et al., 1997), reflecting five fatigue dimensions: lack of energy, physical exertion, physical discomfort, lack of motivation, and sleepiness.

\subsubsection{Electromyography}

Muscle activity of the right Trapezius pars Descendens and Transversus was measured with multichannel surface EMG in a $4 \times 5$ and a $4 \times 7$ grid configuration, respectively, using $\mathrm{Ag} / \mathrm{AgCl}$ surface electrodes (Kendall ${ }^{\mathrm{TM}}$ H69P Cloth Electrodes, Covidien, Zaltbommel, The Netherlands). The electrode rows were placed in line with the muscle fibre directions of both muscles with an inter electrode distance (IED) of $15 \mathrm{~mm}$ (for details, see Luger et al., 2015). Muscle activity of the right Deltoid pars Clavicularis, Acromialis and Spinalis was measured with a bipolar EMG configuration (IED $20 \mathrm{~mm}$ ) according to the Seniam guidelines (Hermens et al., 2000). The skin of the subject was shaved, scrubbed and cleaned with alcohol before electrode attachment. After a visual check of the quality of the raw EMG signals, we started the recordings. EMG was recorded for $10 \mathrm{~s}$ during a reference contraction (Mathiassen et al., 1995) while subjects were holding $1.5 \mathrm{~kg}$ in each hand with the arms in $90^{\circ}$ abduction (frontal plane). EMG recordings lasting for $60 \mathrm{~s}$ were made every second minute during the 60-min experiment. The monopolar EMG signals were online high pass filtered $(5 \mathrm{~Hz})$, amplified with a 128-channel amplifier (REFA, TMS International B.V., Enschede, The Netherlands; input impedance $>10^{12} \Omega$, CMRR $>100 \mathrm{~dB}, 24$ bits $\mathrm{AD}$ conversion after $20 \times$ amplification) and stored on a computer with a sample frequency of $2048 \mathrm{~Hz}$.

Using Matlab (The Mathworks Inc., Natwick, MA, USA), all stored data were offline bipolarly derived, band pass filtered (2nd order Butterworth, 10-400 Hz) and notch filtered (Samani et al., 2009) to remove line interference (2nd order Butterworth, rejection width
$4 \mathrm{~Hz}$ centred at the first seven harmonics of the power line frequency of $50 \mathrm{~Hz}$ ). Two variables were calculated: average rectified value (ARV) and median power frequency (MdPF), two variables commonly used to give an indication of manifestations of muscle fatigue (Basmajian and De Luca, 1985). To calculate the MdPF, we used Welch's periodogram method on the pre-processed data (Welch, 1967). To calculate the ARV, the pre-processed data were rectified and low pass filtered (2nd order Butterworth, $10 \mathrm{~Hz}$ ), creating a linear envelope. We calculated the variability of the EMG amplitude as the median absolute deviation of each one-minute recording (MAD), a robust metric for variability (Chau et al., 2005; Shevlyakov and Vilchevski, 2000). Finally, we calculated the centre of activity of the two Trapezius subparts in medial-lateral (CoA-x) and cranial-caudal direction (CoA-y) according to the method described by Falla and Farina (2007). All EMG amplitudes were normalised to the average amplitude as calculated from the final $9 \mathrm{~s}$ of the reference recording and expressed as percent reference voluntary electrical activation (\%RVE).

\subsection{Data processing and statistical analysis}

Inspection of the data indicated that all variables were approximately normally distributed based on normality tests and visual inspection of histograms, normal Q-Q plots and box plots. For all variables, we calculated the difference between the values at baseline ( $T=0$ for Borg and SOFI; $T=2 \mathrm{~min}$ for EMG) and end ( $T=60 \mathrm{~min})$. To indicate whether we succeeded in designing fatiguing protocols, we performed a One Sample $T$-Test on the difference values between baseline and end. We performed a Paired Samples $T$-Test on all variables to investigate whether task order was present or not (comparing low frequency $S D$ with low frequency $D S)$. To test the effect of rotation frequency and self-selected frequency, we performed a One-Way Repeated Measures Analysis of Variance (ANOVA) with three levels of condition (low, high, selfselected rotation frequency). Significant main effects of condition were further analysed by post hoc Bonferroni-corrected pairwise comparisons. The statistical tests were performed with IBM SPSS Statistics 23.0, using a significance level of $p<0.05$.

\section{Results}

The Paired Sampled T-Tests revealed significant effects of task order on the RPE and physical discomfort increasing more in low frequency $D S$ than in low frequency $S D$, and on the MAD of the M. Trapezius pars Descendens increasing in low frequency $S D$ (8.6\% RVE) but decreasing in low frequency DS ( $-1.2 \% \mathrm{RVE})$. We have merged the two low frequency conditions to exclude the effect of task order.

The experimental protocol was designed to initiate fatigue which was successful for perceived ratings of fatigue in all rotation schedules, with an average increase of 3.7 on the Borg CR-10 $(p<0.05)$. The SOFI fatigue subdivisions, especially lack of energy, physical exertion and physical discomfort significantly increased as signs of fatigue $(p<0.05)$, although not in all rotation schedules (Fig. 3, Table 1).

Fig. 4 presents two-dimensional JASA plots (Joint Analysis of EMG Spectrum and Amplitude; Luttmann et al., 1996) for all muscles. The symbols in the subfigures for each of the rotation schedule are mainly in the lower right quadrant (increase in EMG amplitude accompanied by a decrease in median frequency), which indicates muscle fatigue (Basmajian and De Luca, 1985; Table 1). The development of EMG manifestations of muscle fatigue was significant only in the M. Trapezius pars Descendens for all conditions with an average amplitude increase of $14.3 \%$ RVE and a median frequency decrease of $-4.4 \mathrm{~Hz}(p<0.05)$. We found 


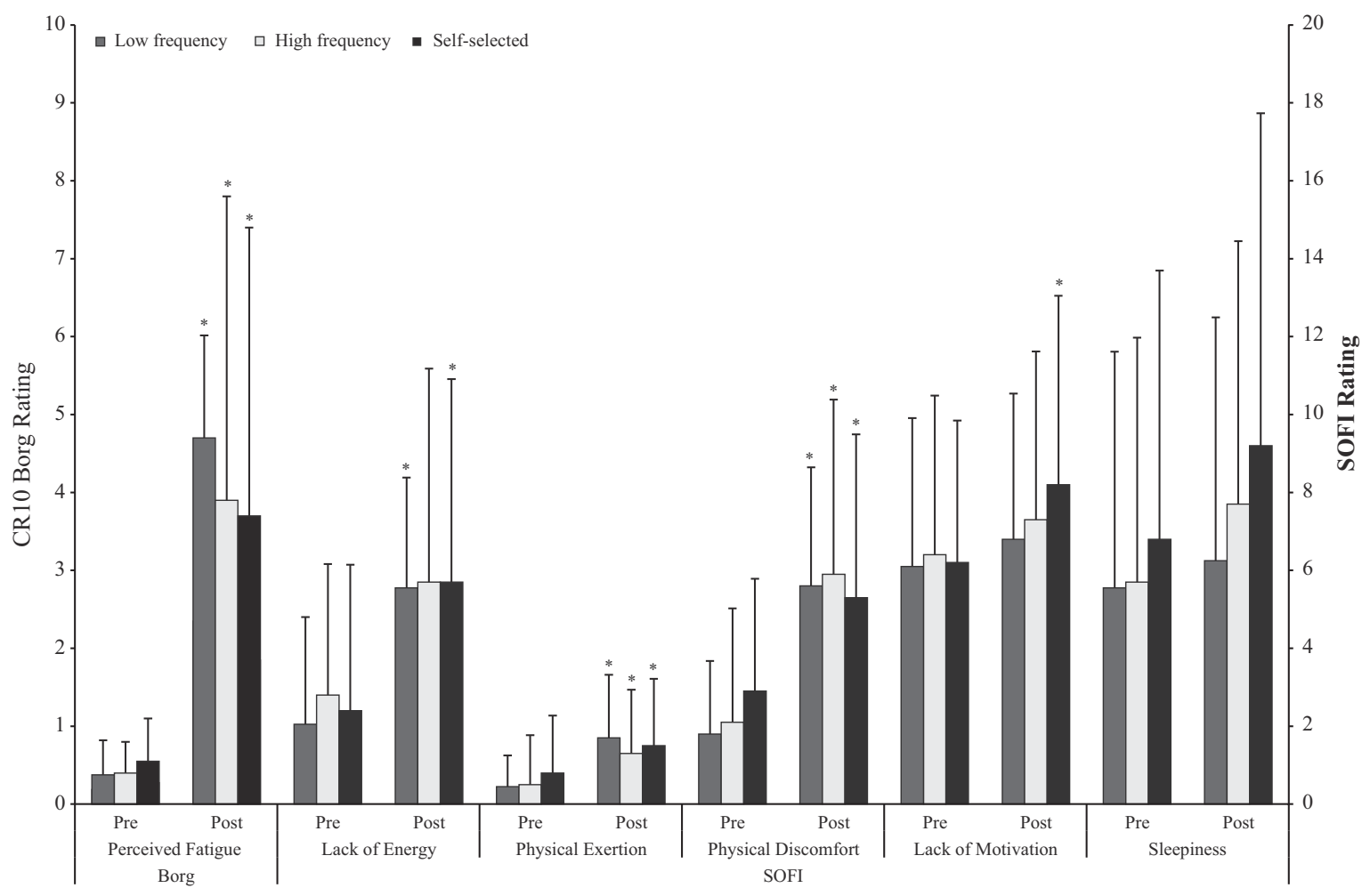

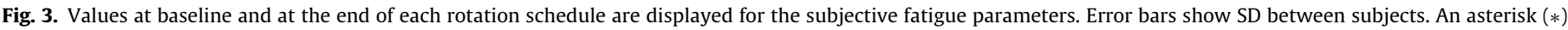
indicates whether the change between the baseline and end values significantly differed from zero $(p<0.05)$.

significant decreases within conditions of the median frequency in the M. Trapezius pars Transversus and Deltoid subparts, without concomitant changes in the amplitudes ( $p<0.05$; Table 1$)$. EMG variability (MAD) of both the Trapezius and Deltoid subparts significantly increased within some rotation schedules $(p<0.05$; Fig. 5). The spatial centre of activity of the M. Trapezius pars Transversus changed only within the low rotation frequency schedule $(p<0.05$; Table 1$)$.

\subsection{Task rotation frequency}

The results of the one-way ANOVAs are presented in Table 2 . For the Borg fatigue rating we found a main effect of condition $(p=0.011)$, after which the pairwise comparisons revealed no effect of rotation frequency (low versus high). The centre of activity of the M. Trapezius pars Descendens showed a main effect of condition for both the medio-lateral $(X)$ and cranial-caudal $(Y)$ directions with significance levels of $p=0.029$ and $p=0.019$, respectively. The post hoc tests indicated that the centre of activity moved medially and cranially in the low rotation frequency, whereas the opposite movement pattern was found for the high rotation frequency. The variability of the M. Deltoid pars Spinalis was also significantly affected by condition $(p=0.004)$, increasing $6.87 \%$ RVE in the high rotation frequency compared to $0.85 \%$ RVE in the low rotation frequency $(p=0.008)$.

\subsection{Self-selected rotation frequency}

In the self-selected rotation schedule, there was not one particular scheme subjects preferred (Fig. 6). On average, we observed that the amount of rotations was 6.7 (SD 3.6).

We found significant main effects of condition for the change of perceived fatigue ( $p=0.011$; Table 2 ). Post hoc tests revealed that the self-selected rotation schedule was perceived as less fatiguing compared to low rotation schemes $(p=0.009)$, while no difference was observed in comparison to high rotation schemes ( $p=1.00$; Table 2). Regarding EMG, we found significant main effects of condition for several variables ( $p<0.05$; Table 2 ), but no significant post hoc pairwise comparisons regarding the self-selected rotation frequency schedule.

\section{Discussion}

The one-hour rotation protocols led to significant signs of fatigue as indicated by perceived fatigue, lack of energy, physical exertion and physical discomfort, and by a combined increase in EMG amplitude and decrease in median frequency of the $M$. Trapezius pars Descendens. EMG variability of both M. Trapezius subparts and the M. Deltoid pars Spinalis changed with fatigue. We aimed to determine the influence of task rotation on fatigue development during the one-hour protocols by rotating between static and dynamic work bouts; however, we found no significant effects of rotation frequency. Furthermore, we aimed to determine the effect of self-selected rotations on fatigue development and found that the self-selected condition was perceived less fatiguing than the low conditions.

\subsection{Rotation frequency}

Task rotation is not a new concept and mainly received its attention as a method possible to increase employee's skills, motivation and productivity (Cheraskin and Campion, 1996; Farrant, 1987). In the past decades, task rotation has also been used as an intervention to increase external exposure variation and to consequently attenuate precursors of musculoskeletal disorders, like manifestations of fatigue (Leider et al., 2015; Straker and Mathiassen, 2009). Theoretically, we expected that task rotation frequency would influence manifestations of fatigue because each 
Table 1

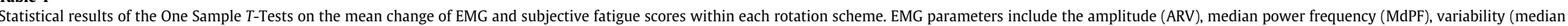
absolute difference; MAD) and centre of activity shifts in the medial-lateral (CoA-x) and cranial-caudal (CoA-y) directions. Significant results $(p<0.05)$ are marked with an asterisk $(*)$.

\begin{tabular}{|c|c|c|c|c|c|c|c|c|c|c|}
\hline & & \multicolumn{3}{|c|}{ Low rotation frequency } & \multicolumn{3}{|c|}{ High rotation frequency } & \multicolumn{3}{|c|}{ Self-selected rotation frequency } \\
\hline & & Mean (SD) & $T(\mathrm{df})$ & $95 \%-\mathrm{CI}$ & Mean (SD) & $T(\mathrm{df})$ & $95 \%-\mathrm{CI}$ & Mean (SD) & $T(\mathrm{df})$ & $95 \%-\mathrm{CI}$ \\
\hline Borg & Fatigue & $4.33(1.22)$ & $11.22(9)$ & {$[3.45 ; 5.20]^{*}$} & $3.50(1.60)$ & $6.92(9)$ & {$[2.36 ; 4.64]^{*}$} & $3.15(1.31)$ & $7.58(9)$ & {$[2.21 ; 4.09]^{*}$} \\
\hline SOFI & $\begin{array}{l}\text { Lack of energy } \\
\text { Physical exertion } \\
\text { Physical discomfort } \\
\text { Lack of motivation } \\
\text { Sleepiness }\end{array}$ & $\begin{array}{l}3.50(2.98) \\
1.25(1.65) \\
3.80(2.49) \\
0.70(1.90) \\
0.70(2.43)\end{array}$ & $\begin{array}{l}3.71(9) \\
2.39(9) \\
4.83(9) \\
1.16(9) \\
0.91(9)\end{array}$ & $\begin{array}{l}{[1.37 ; 5.63]^{*}} \\
{[0.07 ; 2.43]^{*}} \\
{[2.02 ; 5.58]^{*}} \\
{[-0.66 ; 2.06]} \\
{[-1.04 ; 2.44]}\end{array}$ & $\begin{array}{l}2.90(4.18) \\
0.80(0.92) \\
3.80(3.55) \\
0.90(2.23) \\
2.00(6.02)\end{array}$ & $\begin{array}{l}2.20(9) \\
2.75(9) \\
3.38(9) \\
1.27(9) \\
1.05(9)\end{array}$ & $\begin{array}{l}{[-0.09 ; 5.89]} \\
{[0.14 ; 1.46]^{*}} \\
{[1.26 ; 6.34]^{*}} \\
{[-0.70 ; 2.50]} \\
{[-2.31 ; 6.31]}\end{array}$ & $\begin{array}{l}3.30(3.30) \\
0.70(0.95) \\
2.40(1.51) \\
2.00(2.71) \\
2.40(4.70)\end{array}$ & $\begin{array}{l}3.16(9) \\
2.33(9) \\
5.04(9) \\
2.34(9) \\
1.62(9)\end{array}$ & $\begin{array}{l}{[0.94 ; 5.66]^{*}} \\
{[0.02 ; 1.38]^{*}} \\
{[1.32 ; 3.48]^{*}} \\
{[0.06 ; 3.94]^{*}} \\
{[-0.96 ; 5.76]}\end{array}$ \\
\hline M. Trapezius Transversus & $\begin{array}{l}\text { ARV } \\
\text { MdPF } \\
\text { MAD } \\
\text { COA-x } \\
\text { COA-y }\end{array}$ & $\begin{array}{l}-0.18(25.53) \\
-4.95(6.96) \\
0.81(8.14) \\
0.08(0.09) \\
-0.04(0.26)\end{array}$ & $\begin{array}{l}-0.02(9) \\
-2.25(9) \\
0.32(9) \\
2.87(9) \\
-0.50(9)\end{array}$ & $\begin{array}{l}{[-18.44 ; 18.08]} \\
{[-9.93 ; 0.03]} \\
{[-5.01 ; 6.64]} \\
{[0.02 ; 0.15]^{*}} \\
{[-0.23 ; 0.15]}\end{array}$ & $\begin{array}{l}7.73(32.72) \\
-10.57(5.21) \\
6.82(6.32) \\
0.01(0.06) \\
-0.03(0.20)\end{array}$ & $\begin{array}{l}0.71(9) \\
-6.41(9) \\
3.41(9) \\
0.34(9) \\
-0.42(9)\end{array}$ & $\begin{array}{l}{[-16.08 ; 30.73]} \\
{[-14.30 ;-6.84]^{*}} \\
{[2.29 ; 11.34]^{*}} \\
{[-0.03 ; 0.05]} \\
{[-0.17 ; 0.12]}\end{array}$ & $\begin{array}{l}17.73(20.98) \\
-10.93(7.96) \\
8.01(8.06) \\
-0.01(0.15) \\
0.10(0.42)\end{array}$ & $\begin{array}{l}2.67(9) \\
-4.34(9) \\
3.14(9) \\
-0.27(9) \\
0.71(8)\end{array}$ & $\begin{array}{l}{[2.72 ; 32.74]^{*}} \\
{[-16.63 ;-5.23]^{*}} \\
{[2.24 ; 13.77]^{*}} \\
{[-0.13 ; 0.11]} \\
{[-0.22 ; 0.42]}\end{array}$ \\
\hline M. Trapezius Descendens & $\begin{array}{l}\text { ARV } \\
\text { MdPF } \\
\text { MAD } \\
\text { COA-x } \\
\text { COA-y }\end{array}$ & $\begin{array}{l}16.57(15.51) \\
-3.58(2.90) \\
4.32(3.73) \\
-0.04(0.04) \\
0.10(0.10)\end{array}$ & $\begin{array}{l}3.38(9) \\
-3.90(9) \\
3.67(9) \\
-2.97(9) \\
3.07(9)\end{array}$ & $\begin{array}{l}{[5.47 ; 27.66]^{*}} \\
{[-5.65 ;-1.50]^{*}} \\
{[1.66 ; 6.99]^{*}} \\
{[-0.07 ;-0.01]^{*}} \\
{[0.03 ; 0.18]^{*}}\end{array}$ & $\begin{array}{l}17.48(9.60) \\
-5.82(3.24) \\
9.16(4.99) \\
0.00(0.03) \\
-0.01(0.07)\end{array}$ & $\begin{array}{l}5.76(9) \\
-5.67(9) \\
5.80(9) \\
0.40(9) \\
-0.49(9)\end{array}$ & $\begin{array}{l}{[10.61 ; 24.35]^{*}} \\
{[-8.13 ;-3.50]^{*}} \\
{[5.59 ; 12.73]} \\
{[-0.02 ; 0.02]} \\
{[-0.06 ; 0.04]}\end{array}$ & $\begin{array}{l}8.88(10.94) \\
-3.91(3.16) \\
5.86(4.91) \\
0.00(0.05) \\
-0.00(0.09)\end{array}$ & $\begin{array}{l}2.57(9) \\
-3.92(9) \\
3.78(9) \\
0.07(8) \\
-0.05(8)\end{array}$ & $\begin{array}{l}{[1.05 ; 16.71]^{*}} \\
{[-6.17 ;-1.66]^{*}} \\
{[2.35 ; 9.37]^{*}} \\
{[-0.03 ; 0.04]} \\
{[-0.07 ; 0.07]}\end{array}$ \\
\hline M. Deltoid Clavicularis & $\begin{array}{l}\text { ARV } \\
\text { MdPF } \\
\text { MAD }\end{array}$ & $\begin{array}{l}0.26(3.82) \\
-3.00(8.66) \\
2.93(9.03)\end{array}$ & $\begin{array}{l}0.20(8) \\
-1.10(9) \\
1.02(9)\end{array}$ & $\begin{array}{l}{[-2.68 ; 3.19]} \\
{[-9.19 ; 3.19]} \\
{[-3.53 ; 9.39]}\end{array}$ & $\begin{array}{l}2.52(8.46) \\
-14.80(9.35) \\
0.34(2.31)\end{array}$ & $\begin{array}{l}0.94(9) \\
-5.00(9) \\
0.46(9)\end{array}$ & $\begin{array}{l}{[-3.53 ; 8.57]} \\
{[-21.49 ;-8.11]^{*}} \\
{[-1.31 ; 1.99]}\end{array}$ & $\begin{array}{l}10.36(28.20) \\
-10.70(9.70) \\
2.51(7.13)\end{array}$ & $\begin{array}{l}1.16(9) \\
-3.49(9) \\
1.11(9)\end{array}$ & $\begin{array}{l}{[-9.81 ; 30.54]} \\
{[-17.64 ;-3.76]^{*}} \\
{[-2.59 ; 7.61]}\end{array}$ \\
\hline M. Deltoid Acromialis & $\begin{array}{l}\text { ARV } \\
\text { MdPF } \\
\text { MAD }\end{array}$ & $\begin{array}{l}0.55(4.13) \\
-5.00(3.25) \\
-0.51(2.79)\end{array}$ & $\begin{array}{l}0.42(9) \\
-4.87(9) \\
-0.58(9)\end{array}$ & $\begin{array}{l}{[-2.41 ; 3.51]} \\
{[-7.32 ;-2.68]^{*}} \\
{[-2.50 ; 1.49]}\end{array}$ & $\begin{array}{l}-0.43(8.17) \\
-11.00(6.68) \\
0.46(3.10)\end{array}$ & $\begin{array}{l}-0.17(9) \\
-5.21(9) \\
0.47(9)\end{array}$ & $\begin{array}{l}{[-6.27 ; 5.41]} \\
{[-15.78 ;-6.22]^{*}} \\
{[-1.75 ; 2.68]}\end{array}$ & $\begin{array}{l}3.47(6.23) \\
-9.20(5.25) \\
1.18(1.88)\end{array}$ & $\begin{array}{l}1.76(9) \\
-5.55(9) \\
1.99(9)\end{array}$ & $\begin{array}{l}{[-0.98 ; 7.93]} \\
{[-12.95 ;-5.45]^{*}} \\
{[-0.16 ; 2.52]}\end{array}$ \\
\hline M. Deltoid Spinalis & $\begin{array}{l}\text { ARV } \\
\text { MdPF } \\
\text { MAD }\end{array}$ & $\begin{array}{l}1.48(5.69) \\
-6.45(5.93) \\
0.85(1.85)\end{array}$ & $\begin{array}{l}0.82(9) \\
-3.44(9) \\
1.45(9)\end{array}$ & $\begin{array}{l}{[-2.59 ; 5.55]} \\
{[-10.69 ;-2.21]^{*}} \\
{[-0.48 ; 2.18]}\end{array}$ & $\begin{array}{l}7.65(13.87) \\
-4.90(6.61) \\
6.87(4.39)\end{array}$ & $\begin{array}{l}1.75(9) \\
-2.35(9) \\
4.95(9)\end{array}$ & $\begin{array}{l}{[-2.26 ; 17.57]} \\
{[-9.63 ;-0.17]^{*}} \\
{[3.73 ; 10.01]^{*}}\end{array}$ & $\begin{array}{l}5.46(7.43) \\
-5.50(.25) \\
4.48(4.96)\end{array}$ & $\begin{array}{l}2.33(9) \\
-4.09(9) \\
2.86(9)\end{array}$ & $\begin{array}{l}{[0.15 ; 10.78]^{*}} \\
{[-8.54 ;-2.46]^{*}} \\
{[0.93 ; 8.02]^{*}}\end{array}$ \\
\hline
\end{tabular}



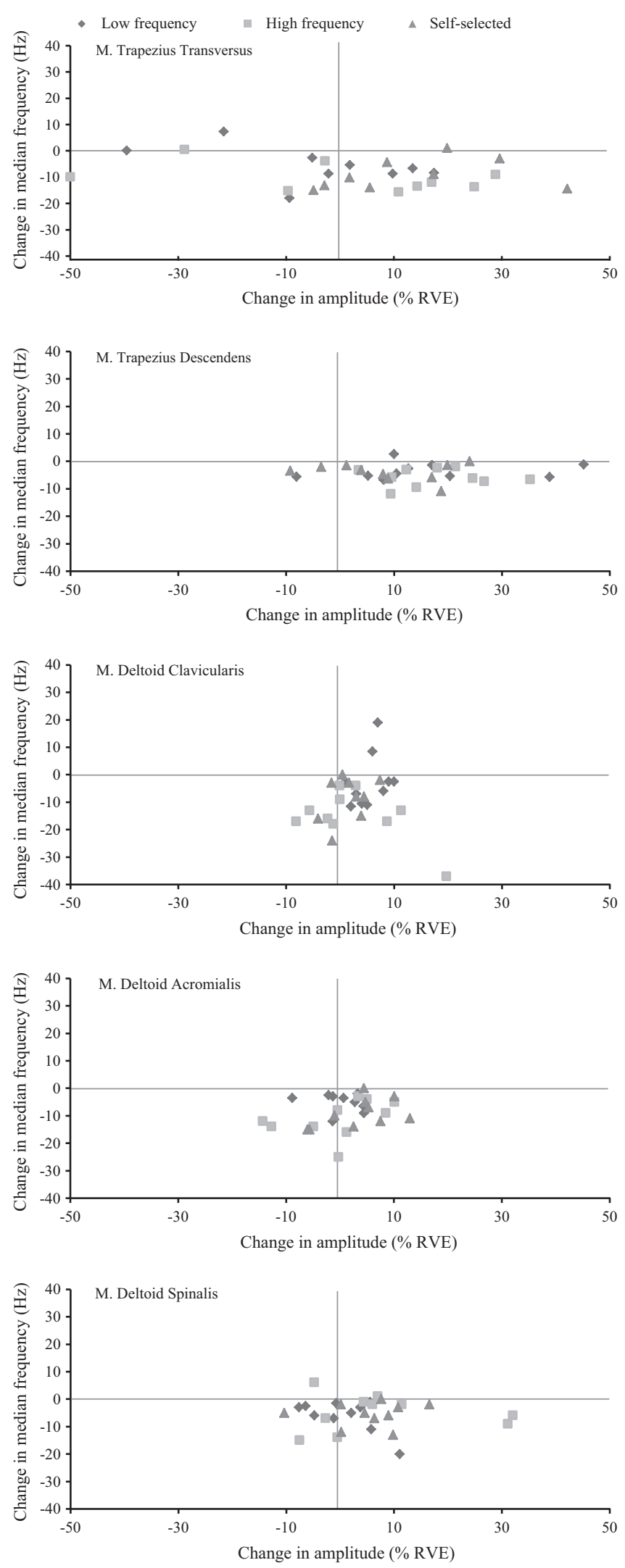

Fig. 4. Two-dimensional JASA plots for EMG amplitude and median frequency changes within the rotation schedules (end at $T=60$ min minus baseline at $T=2 \mathrm{~min}$ ) for all muscles and participants.

rotation provides an opportunity for the muscle to recover from its continuous loading (Howarth et al., 2009; Mathiassen, 2006). Furthermore, we expected that rotating static and dynamic work would create even better opportunities for muscle recovery because dynamic work on itself may enhance load sharing between muscles, have additional motor unit recruitment, and allow for greater blood perfusion to the muscles (e.g., Frey Law et al., 2010). Arguing the other way around, blood perfusion may be restricted during static work, resulting in arterial vasodilatation and produce pain producing substances (such as nitric oxide) and inflammatory mediators (such as histamine; Knardahl, 2002; Visser and van Dieën, 2006), which activate nociceptors and create sensations of discomfort (Frey Law et al., 2008; Shah et al., 2005; Simons, 2004). Our hypothesis was not confirmed by any of our findings. For the Borg and SOFI ratings, this may imply that the two tasks did not differ enough from each other to significantly decrease with a higher rotation frequency. Regarding our EMG parameters, which only showed significant manifestations of muscle fatigue in the M. Trapezius pars Descendens, we can argue that either the tasks did not differ enough in muscle loading or the one-hour protocols were not fatiguing enough. The latter explanation seems not very likely, since the Borg and SOFI ratings reveal clear manifestations of fatigue (Fig. 3). Previous studies investigating the effect of rotation frequency between lifting of $10 \%$ and $20 \%$ body weight (Horton et al., 2015), shoulder flexion and abduction (Raina and Dickerson, 2009), and static shoulder exertions at 15\% and 30\% MVC (Horton et al., 2012) on manifestations of muscle fatigue and perceived fatigue are in line with our findings. Because we clearly saw more development of perceived fatigue in the low compared to the high rotation frequency protocols, the two experimental tasks may not have been different enough in terms of muscle activation patterns, or the effects of the rotation schemes may not have been present yet within $1 \mathrm{~h}$.

We considered the EMG variability and its spatial centre of activity parameters because they could be influenced by manifestations of muscle fatigue. In their review, Srinivasan and Mathiassen (2012) indicate that slower fatigue development may be due to more motor variability and indicate that this association may be bi-directional. Table 1 mostly shows us that whenever the muscle's activity increases, so does its variability and vice versa. These results tend to the regrettable situation pointing out that variability may be a consequence of manifestations of muscle fatigue rather than a prevention mechanism to avoid (further) manifestations of muscle fatigue. Another parameter which may reflect our ability to change between motor strategies, is said to be a shift in the spatial centre of activity of a muscle (Falla and Farina, 2007). We did identify a significant shift of the centre of activity in the M. Trapezius pars Descendens more towards cranial and medial in the low compared to the high rotation frequency. This makes it even more difficult to elaborate on the directionality of the association between motor variability and muscular fatigue development.

\subsection{Autonomy}

Although imposed task rotation schedules may lead to increased employee's motivation and productivity, it does not necessarily influence the degree of autonomy an employee has during work. The freedom of choosing a preferred rotation pattern, which may reflect sort of a non-standardised psychophysical approach, confirmed our expectation that the self-selected protocol resulted in less development of perceived fatigue compared to the low and high protocols. This finding is in line with the results of Kraatz et al. (2013), who showed that autonomy is negatively related to fatigue development. Our finding also agrees with the theory as presented in the classical Karasek model, which assumes that high levels of job demands with low job control will lead to symptoms of stress which may increase the risks to, for example, cardiovascular disorders (Karasek, 1979). As Borg (1982) already 


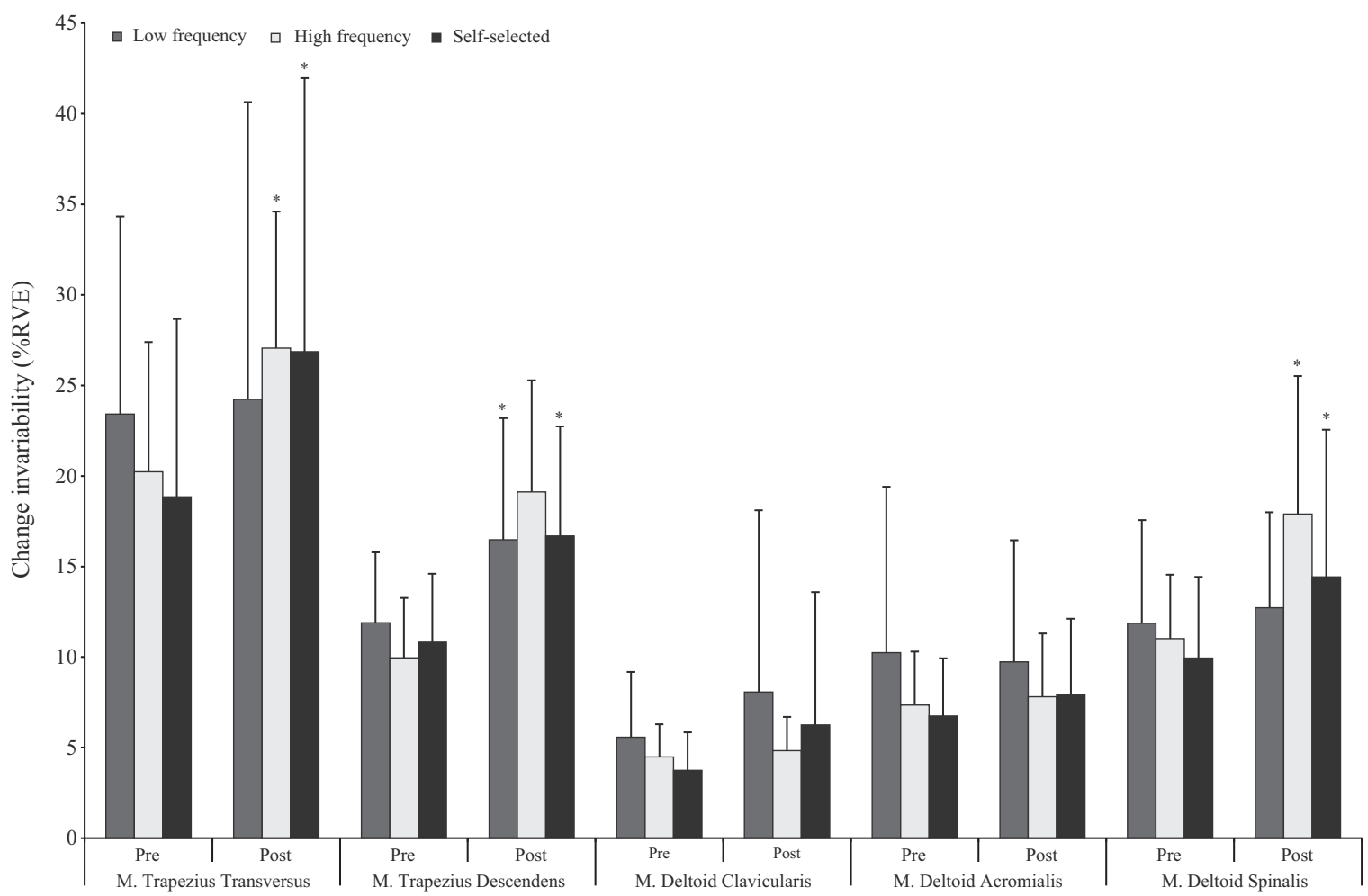

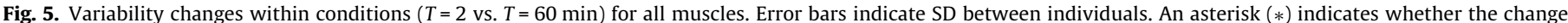
from the baseline and end values significantly differed from zero $(p<0.05)$.

Table 2

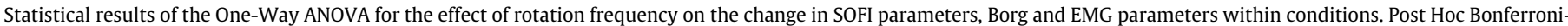

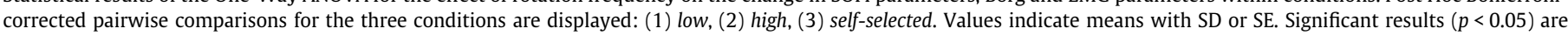
marked with an asterisk $(*)$.

\begin{tabular}{|c|c|c|c|c|c|c|c|c|c|}
\hline & & \multicolumn{5}{|l|}{ Condition } & \multicolumn{3}{|c|}{ Pairwise comparisons } \\
\hline & & Low (SE) & High (SE) & Self (SE) & $F(\mathrm{df})$ & $p$-value & Low vs. high & Low vs. self & High vs. self \\
\hline Borg & Fatigue & $4.33(0.39)$ & $3.50(0.51)$ & $3.15(0.42)$ & $5.90(2)$ & $0.011^{*}$ & 0.213 & $0.009^{*}$ & 1.000 \\
\hline SOFI & $\begin{array}{l}\text { Lack of energy } \\
\text { Physical exertion } \\
\text { Physical discomfort } \\
\text { Lack of motivation } \\
\text { Sleepiness }\end{array}$ & $\begin{array}{l}3.50(0.94) \\
1.25(0.52) \\
3.80(0.79) \\
0.70(0.60) \\
0.70(0.77)\end{array}$ & $\begin{array}{l}2.90(1.32) \\
0.80(0.29) \\
3.80(1.12) \\
0.90(0.71) \\
2.00(1.90)\end{array}$ & $\begin{array}{l}3.30(1.04) \\
0.70(0.30) \\
2.40(0.48) \\
2.00(0.86) \\
2.40(1.49)\end{array}$ & $\begin{array}{l}0.20(2) \\
0.90(2) \\
2.19(2) \\
1.31(2) \\
0.51(2)\end{array}$ & $\begin{array}{l}0.822 \\
0.417 \\
0.140 \\
0.294 \\
0.611\end{array}$ & $\begin{array}{l}1.000 \\
1.000 \\
1.000 \\
1.000 \\
1.000\end{array}$ & $\begin{array}{l}1.000 \\
0.600 \\
0.110 \\
0.795 \\
0.614\end{array}$ & $\begin{array}{l}1.000 \\
1.000 \\
0.332 \\
0.412 \\
1.000\end{array}$ \\
\hline M. Trapezius Transversus & $\begin{array}{l}\text { ARV } \\
\text { MdPF } \\
\text { MAD } \\
\text { COA-x } \\
\text { COA-y }\end{array}$ & $\begin{array}{l}-0.18(8.07) \\
-4.95(2.20) \\
0.81(2.58) \\
0.07(0.03) \\
-0.02(0.09)\end{array}$ & $\begin{array}{l}7.33(10.35) \\
-10.57(1.65) \\
6.82(2.00) \\
0.00(0.02) \\
-0.01(0.07)\end{array}$ & $\begin{array}{l}17.73(6.64) \\
-10.93(2.52) \\
8.01(2.55) \\
-0.01(0.05) \\
0.10(0.14)\end{array}$ & $\begin{array}{l}1.38(2) \\
2.23(2) \\
2.66(2) \\
1.68(2) \\
0.40(1.21)\end{array}$ & $\begin{array}{l}0.277 \\
0.137 \\
0.098 \\
0.217 \\
0.578\end{array}$ & $\begin{array}{l}1.000 \\
0.440 \\
0.455 \\
0.204 \\
1.000\end{array}$ & $\begin{array}{l}0.137 \\
0.193 \\
0.069 \\
0.639 \\
1.000\end{array}$ & $\begin{array}{l}1.000 \\
1.000 \\
1.000 \\
1.000 \\
1.000\end{array}$ \\
\hline M. Trapezius Descendens & $\begin{array}{l}\text { ARV } \\
\text { MdPF } \\
\text { MAD } \\
\text { COA-x } \\
\text { COA-y }\end{array}$ & $\begin{array}{l}16.57(4.91) \\
-3.58(0.92) \\
4.32(1.18) \\
-0.05(0.01) \\
0.12(0.03)\end{array}$ & $\begin{array}{l}17.48(3.04) \\
-5.82(1.03) \\
9.16(1.58) \\
0.00(0.01) \\
-0.01(0.02)\end{array}$ & $\begin{array}{l}8.88(3.46) \\
-3.91(1.00) \\
5.86(1.55) \\
0.00(0.02) \\
-0.00(0.03)\end{array}$ & $\begin{array}{l}1.87(2) \\
1.50(2) \\
3.88(2) \\
6.41(1.15) \\
7.62(1.17)\end{array}$ & $\begin{array}{l}0.183 \\
0.250 \\
0.060 \\
0.029^{*} \\
0.019^{*}\end{array}$ & $\begin{array}{l}1.000 \\
0.532 \\
0.004^{*} \\
0.036^{*} \\
0.031^{*}\end{array}$ & $\begin{array}{l}0.671 \\
1.000 \\
1.000 \\
0.148 \\
0.103\end{array}$ & $\begin{array}{l}0.131 \\
0.695 \\
0.434 \\
1.000 \\
1.000\end{array}$ \\
\hline M. Deltoid Clavicularis & $\begin{array}{l}\text { ARV } \\
\text { MdPF } \\
\text { MAD }\end{array}$ & $\begin{array}{l}0.26(1.27) \\
-3.00(2.74) \\
2.93(2.86)\end{array}$ & $\begin{array}{l}0.61(2.09) \\
-14.80(2.96) \\
0.34(0.73)\end{array}$ & $\begin{array}{l}1.51(1.19) \\
-10.70(3.07) \\
2.51(2.25)\end{array}$ & $\begin{array}{l}0.17(2) \\
4.17(1.27) \\
1.12(1.02)\end{array}$ & $\begin{array}{l}0.849 \\
0.058 \\
0.318\end{array}$ & $\begin{array}{l}1.000 \\
0.136 \\
0.992\end{array}$ & $\begin{array}{l}1.000 \\
0.390 \\
1.000\end{array}$ & $\begin{array}{l}1.000 \\
0.250 \\
0.819\end{array}$ \\
\hline M. Deltoid Acromialis & $\begin{array}{l}\text { ARV } \\
\text { MdPF } \\
\text { MAD }\end{array}$ & $\begin{array}{l}0.55(1.31) \\
-5.00(1.03) \\
-0.51(0.88)\end{array}$ & $\begin{array}{l}-0.43(2.58) \\
-11.00(2.11) \\
0.46(0.98)\end{array}$ & $\begin{array}{l}3.48(1.97) \\
-9.20(1.66) \\
1.18(0.59)\end{array}$ & $\begin{array}{l}3.44(2) \\
4.76(2) \\
1.35(2)\end{array}$ & $\begin{array}{l}0.054 \\
0.022^{*} \\
0.285\end{array}$ & $\begin{array}{l}1.000 \\
0.124 \\
1.000\end{array}$ & $\begin{array}{l}0.277 \\
0.100 \\
0.559\end{array}$ & $\begin{array}{l}0.010^{*} \\
0.924 \\
1.000\end{array}$ \\
\hline M. Deltoid Spinalis & $\begin{array}{l}\text { ARV } \\
\text { MdPF } \\
\text { MAD }\end{array}$ & $\begin{array}{l}1.48(1.80) \\
-6.45(1.88) \\
0.85(0.59)\end{array}$ & $\begin{array}{l}7.65(4.39) \\
-4.90(2.09) \\
6.87(1.39)\end{array}$ & $\begin{array}{l}5.46(2.35) \\
-5.50(1.34) \\
4.48(1.57)\end{array}$ & $\begin{array}{l}2.00(2) \\
0.18(2) \\
7.52(2)\end{array}$ & $\begin{array}{l}0.164 \\
0.838 \\
0.004^{*}\end{array}$ & $\begin{array}{l}0.374 \\
1.000 \\
0.008^{*}\end{array}$ & $\begin{array}{l}0.403 \\
1.000 \\
0.283\end{array}$ & $\begin{array}{l}1.000 \\
1.000 \\
0.227\end{array}$ \\
\hline
\end{tabular}

introduced that the perceived ratings of exertion, discomfort and fatigue are reflecting the psychophysical state of a person, our findings may, therefore, not be surprising.
Perceived fatigue results were not supported by EMG indicators of fatigue, although we found a tendency for a cranial shift of the spatial centre of activity in the standardised protocols compared 


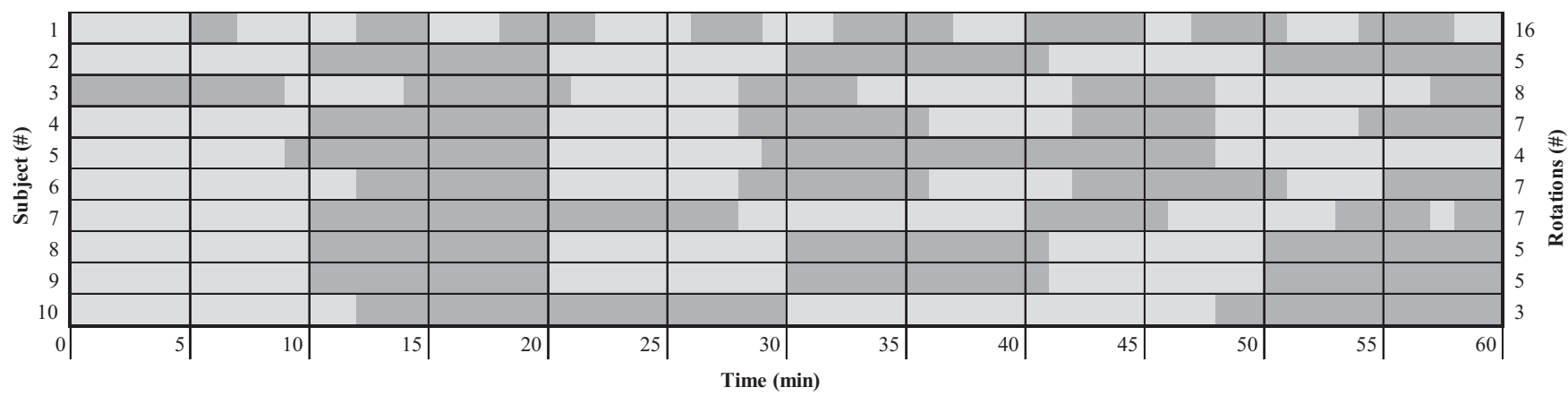

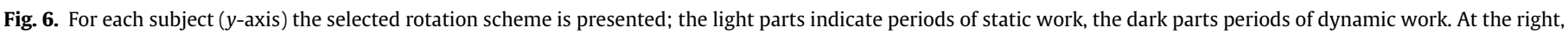
the number of rotations is presented.

to a slight caudal shift of the spatial centre of activity in the self-selected protocol. The cranial shift in the standardised protocols might reflect the tendency to elevate the shoulder complex in order to relief the constantly active shoulder muscle parts (e.g., Luger et al., 2015; Samani et al., 2010). This explanation may support the psychophysical findings somewhat, as reported in the previous paragraph.

\subsection{Task order}

We were not able to identify significant differences between the low and high rotation frequency protocols. However, we did observe some interesting findings with respect to the two low rotation frequency conditions, which we merged within this study. Both low rotation frequency protocols were performed with a different task order. When subjects performed the dynamic task during the first $30 \mathrm{~min}$ and performed the static task during the final $30 \mathrm{~min}$, they arrived at a larger rate of perceived exertion (5.5 [SD 1.9]) compared to performing the protocol in the opposite order (3.1 [SD 1.5]). This may have led to the rotation choice subjects made in the self-selected protocol: nine out of our ten subjects selected to start with the static task while eight out of ten preferred to end with the dynamic task. The EMG indicators of fatigue did not show differences with regard to task order. Thus, based on perceived fatigue, one could argue that task order may be crucial after all, although we cannot directly relate it to the conclusion of Raina and Dickerson (2009) and Horton et al. (2012) that starting with the more difficult or intense of two tasks results in higher levels of perceived fatigue. On the other hand, based on the EMG indicators of fatigue it seems that order of rotation may not be a very important factor (Keir et al., 2011). All in all, this study was not designed to make a statement about task order and our results do not give clear indications to advice on the order manual handling tasks should be performed.

\subsection{Strengths and limitations}

In line with previous lab studies, our results clearly point out that perceived fatigue is much more sensitive to explore whether situations may be physically or mentally harmful than EMG manifestations of muscle fatigue and one should be careful with the reliability of EMG measurements during relatively low-intensity work (10-25\%MVC; Movahed et al., 2011). Even more caution is required when elaborating on the cause-effect relationship between manifestations of muscle fatigue and EMG parameters (Bosch et al., 2012), because our results tend more towards the explanation that EMG variability merely is a result of increased manifestations of muscle fatigue than it is a preventive mechanism for (further) manifestations of muscle fatigue.
When extrapolating the outcomes of this study, one must remember that the nature of work and workplaces varies substantially which makes studies or practical examples not always easily comparable (Keir et al., 2011). Our choice to present the selfselected protocol always the last has the advantage that all subjects, which were no experts in manual materials handling, have a similar reference frame to choose a rotation scheme; however, the disadvantage may be a potential learning effect of fatigue bias due to this choice of order. Furthermore, the small population in this study consisted of healthy young adults and may not be representative for the working population. Gender may have influenced the results because males and females differ in their muscle architecture and consequently muscle recruitment patterns (Côté, 2012). Another important aspect is the fact that not every work situation has the opportunity to apply task rotation or may benefit from task rotation when pacing or quality get critically affected. This not only emphasises the importance of risks on human beings but also on the company's economic prosperity.

\section{Conclusion}

Although we could not find any significant effect of rotation frequency on fatigue development with regard to the standardised protocols, the self-selected protocol, where subjects on average rotated 6.2 times/h (SD 3.6), clearly led to less development of perceived fatigue than standardised protocols. This result first indicates that freedom to choose for the number of rotations may influence the subjective ratings of fatigue more than does an imposed rotation frequency, and second means that letting the employee free in performing work may give the best opportunities to lower the risk to develop musculoskeletal disorders. These findings give reason to more extensively investigate the role of autonomy in a more standardised psychophysical protocol in the search for optimal external exposure variation.

\section{Conflicts of interest}

The authors declare that there are no conflicts of interest.

\section{Acknowledgments}

The authors would like to thank technicians Léon Schutte, Frans-Jozef Halkes, Siro Otten and Hans Agricola for helping with the task set-up and giving technical support during the (preparations of the) measurements. We also would like to thank Pablo Ortega Auriol for his help in the execution of the measurements. 


\section{References}

Åhsberg, E., Gamberale, F., Kjellberg, A., 1997. Perceived quality of fatigue during different occupational tasks - development of a questionnaire. Int. J. Ind. Ergon. 20 (2), 121-135.

Basmajian, J., De Luca, C., 1985. Muscles Alive: Their Functions Revealed by Electromyography, fifth ed. Lippincott, Williams and Wilkins, Baltimore.

Bernard, B., 1997. Musculoskeletal Disorders and Workplace Factors: A Critical Review of Epidemiologic Evidence for Work-Related Musculoskeletal Disorders of the Neck, Upper Extremity, and Low Back. NIOSH, Cincinnati.

Borg, G., 1982. Psychophysical bases of perceived exertion. Med. Sci. Sports Exerc. 14 (5), 377-381.

Bosch, T., Mathiassen, S.E., Hallman, D., de Looze, M.P., Lyskov, E., Visser, B., et al., 2012. Temporal strategy and performance during a fatiguing short-cycle repetitive task. Ergonomics 55 (8), 863-873.

Chau, T., Young, S., Redekop, S., 2005. Managing variability in the summary and comparison of gait data. J. Neuroeng. Rehabil. 2, 22.

Cheraskin, L., Campion, M.A., 1996. Study clarifies job-rotation benefits. Pers. J. 75 (11), 31-38.

Christensen, H., Søgaard, K., Jensen, B.R., Finsen, L., Sjøgaard, G., 1995. Intramuscular and surface EMG power spectrum from dynamic and static contractions. J. Electromyogr. Kinesiol. 5 (1), 27-36.

Côté, J.N., 2012. A critical review on physical factors and functional characteristics that may explain a sex/gender difference in work-related neck/shoulder disorders. Ergonomics 55 (2), 173-182.

Falla, D., Farina, D., 2007. Periodic increases in force during sustained contraction reduce fatigue and facilitate spatial redistribution of trapezius muscle activity. Exp. Brain Res. 182 (1), 99-107.

Farrant, A., 1987. Job rotation is important. Supervision 49 (8), 14-16.

Frey Law, L.A., Lee, J.E., McMullen, T.R., Xia, T., 2010. Relationships between maximum holding time and ratings of pain and exertion differ for static and dynamic tasks. Appl. Ergon. 42 (1), 9-15.

Frey Law, L.A., Sluka, K.A., McMullen, T., Lee, J., Arendt-Nielsen, L., Graven-Nielsen, T., 2008. Acidic buffer induced muscle pain evokes referred pain and mechanical hyperalgesia in humans. Pain 140 (2), 254-264.

Genaidy, A.M., Houshyar, A., Asfour, S.S., 1990. Physiological and psychophysical responses to static dynamic and combined arm tasks. Appl. Ergon. 21 (1), 63-67.

Hermens, H.J., Freriks, B., Disselhorst-Klug, C., Rau, G., 2000. Development of recommendations for SEMG sensors and sensor placement procedures. J. Electromyogr. Kinesiol. 10 (5), 361-374.

Horton, L.M., Nussbaum, M.A., Agnew, M.J., 2012. Effects of rotation frequency and task order on localised muscle fatigue and performance during repetitive static shoulder exertions. Ergonomics 55 (10), 1205-1217.

Horton, L.M., Nussbaum, M.A., Agnew, M.J., 2015. Rotation during lifting tasks: effects of rotation frequency and task order on localized muscle fatigue and performance. J. Occup. Environ. Hyg. 12 (2), 95-106.

Howarth, S.J., Beach, T.A.C., Pearson, A.J., Callaghan, J.P., 2009. Using sitting as a component of job rotation strategies: are lifting/lowering kinetics and kinematics altered following prolonged sitting. Appl. Ergon. 40 (3), 433-439.

Jonsson, B., 1988. Electromyographic studies of job rotation. Scand. J. Work Environ. Health 14 (Suppl. 1), 108-109.

Karasek, R.A., 1979. Job demands, job decision latitude and mental strain implications for job redesign. Adm. Sci. Q. 24 (2), 285-308.

Keir, P.J., Sanei, K., Holmes, M.W.R., 2011. Task rotation effects on upper extremity and back muscle activity. Appl. Ergon. 42 (6), 814-819.

Knardahl, S., 2002. Psychophysiological mechanisms of pain in computer work: the blood vessel-nociceptor interaction hypothesis. Work Stress 16 (2), 179-189.

Kraatz, S., Lang, J., Kraus, T., Münster, E., Ochsmann, E., 2013. The incremental effect of psychosocial workplace factors on the development of neck and shoulder disorders: a systematic review of longitudinal studies. Int. Arch. Occup. Environ. Health 86 (4), 375-395.

Kuijer, P.P.F.M., van der Beek, A.J., van Dieën, J.H., Visser, B., Frings-Dresen, M.H.W. 2005. Effect of job rotation on need for recovery, musculoskeletal complaints, and sick leave due to musculoskeletal complaints: a prospective study among refuse collectors. Am. J. Ind. Med. 47 (5), 394-402.

Kuijer, P.P.F.M., Visser, B., Kemper, H.C.G., 1999. Job rotation as a factor in reducing physical workload at a refuse collecting department. Ergonomics 42 (9), 1167-1178.

Kuijer, P.P.F.M., Vries, W.H.K., Beek, A.J., Dieën, J.H., Visser, B., Frings-Dresen, M.H. W., 2004. Effect of job rotation on work demands, workload, and recovery of refuse truck drivers and collectors. Hum. Factors 46 (3), 437-448.

Larsson, B., Søgaard, K., Rosendal, L., 2007. Work related neck-shoulder pain: a review on magnitude, risk factors, biochemical characteristics, clinical picture and preventive interventions. Best Pract. Res. Clin. Rheumatol. 21 (3), 447-463.

Leider, P.C., Boschman, J.S., Frings-Dresen, M.H., van der Molen, H.F., 2015. Effects of job rotation on musculoskeletal complaints and related work exposures: a systematic literature review. Ergonomics 58 (1), 18-32.

Linaker, C.H., Walker-Bone, K., 2015. Shoulder disorders and occupation. Best Pract. Res. Clin. Rheumatol. 29 (3).

Luger, T., Bosch, T., Hoozemans, M., de Looze, M., Veeger, D., 2015. Task variation during simulated, repetitive, low-intensity work - influence on manifestation of shoulder muscle fatigue, perceived discomfort and upper-body postures. Ergonomics 58 (11), 1851-1867.

Luttmann, A., Sökeland, J., Laurig, W., 1996. Electromyographical study on surgeons in urology. I. Influence of the operating technique on muscular strain Ergonomics 39 (2), 285-297.

Madeleine, P., 2010. On functional motor adaptations: from the quantification of motor strategies to the prevention of musculoskeletal disorders in the neck-shoulder region. Acta Physiol. 199 (Suppl. 679), 1-46.

Mathiassen, S.E., 2006. Diversity and variation in biomechanical exposure: what is it, and why would we like to know? Appl. Ergon. 37 (4), 419-427.

Mathiassen, S.E., Winkel, J., Hägg, M., 1995. Normalization of surface EMC amplitude from the upper trapezius muscle in ergonomics - a review. J. Electromyogr. Kinesiol. 5 (4), 197-226.

Movahed, M., Ohashi, J.Y., Kurustien, N., Izumi, H., Kumashiro, M., 2011. Fatigue sensation, electromyographical and hemodynamic changes of low back muscles during repeated static contraction. Eur. J. Appl. Physiol. 111 (3), 459-467.

Raina, S.M., Dickerson, C.R., 2009. The influence of job rotation and task order on muscle fatigue - a deltoid example. Work 34 (2), 205-213.

Rashedi, E., Nussbaum, M.A., 2015. A review of occupationally-relevant models of localised muscle fatigue. Int. J. Hum. Factors Model. Simul. 5 (1), 61-80.

Samani, A., Holtermann, A., Sogaard, K., Madeleine, P., 2009. Active pauses induce more variable electromyographic pattern of the trapezius muscle activity during computer work. J. Electromyogr. Kinesiol. 19 (6), e430-e437.

Samani, A., Holtermann, A., Søgaard, K., Madeleine, P., 2010. Active biofeedback changes the spatial distribution of upper trapezius muscle activity during computer work. Eur. J. Appl. Physiol. 110 (2), 415-423.

Shah, J.P., Phillips, T.M., Danoff, J.V., Gerber, L.H., 2005. An in vivo microanalytical technique for measuring the local biochemical milieu of human skeletal muscle. J. Appl. Physiol. (1985) 99 (5), 1977-1984.

Shevlyakov, G.L., Vilchevski, N.O., 2000. Robustness in Data Analysis. St. Petersburg State Technical University, St. Petersburg.

Simons, D.G., 2004. Review of enigmatic MTrPs as a common cause of enigmatic musculoskeletal pain and dysfunction. J. Electromyogr. Kinesiol. 14 (1), 95-107.

Søgaard, K., 1995. Motor unit recruitment pattern during low-level static and dynamic contractions. Muscle Nerve 18 (3), 292-300.

Srinivasan, D., Mathiassen, S.E., 2012. Motor variability in occupational health and performance. Clin. Biomech. 27 (10), 979-993.

Straker, L., Mathiassen, S.E., 2009. Increased physical work loads in modern work - a necessity for better health and performance? Ergonomics 52 (10), 1215-1225.

Visser, B., van Dieën, J.H., 2006. Pathophysiology of upper extremity muscle disorders. J. Electromyogr. Kinesiol. 16 (1), 1-16.

Welch, P.D., 1967. The use of fast Fourier transform for the estimation of power spectra: a method based on time averaging over short, modified periodograms. IEEE Trans. Audio Electroacoust. 15 (2), 70-73.

Wu, G., van der Helm, F.C.T., Veeger, H.E.J., Makhsous, M., van Roy, P., Anglin, C. et al., 2005. ISB recommendation on definitions of joint coordinate systems of various joints for the reporting of human joint motion - Part II: shoulder, elbow, wrist and hand. J. Biomech. 38 (5), 981-992.

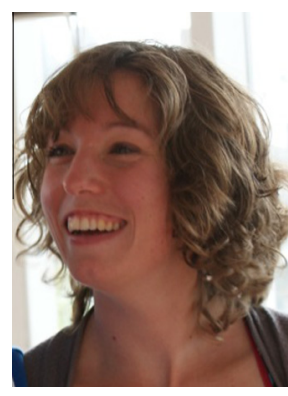

Tessy Luger obtained a M.Sc. degree in Human Movement Sciences in 2011 at the Vrije Universiteit Amsterdam. She started her Ph.D. in 2012 at the Vrije Universiteit in Amsterdam and is supervised by a team of experts from both the Vrije Universiteit Amsterdam and TNO Leiden. The thesis is about task variation during low-intensity hand-arm work. Since 2013, she works as an editor for the Dutch Journal of Human Factors (Tijdschrift voor Human Factors).

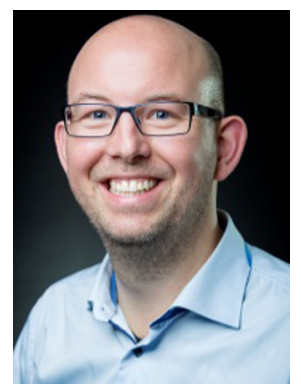

Tim Bosch received his M.Sc. degree in Human Movement Sciences in 2003 from the Vrije Universiteit Amsterdam. He got his Ph.D. in 2011. Since 2003, he is working at TNO (Netherlands Institute for Applied Research) as a researcher focusing on applied industrial research projects. Currently, his main research interests are on industrial ergonomics, effects of physical workload and fatigue on productivity and the effects of production automation on human operators. He was (co-)author of over 20 full papers in international scientific journals. 


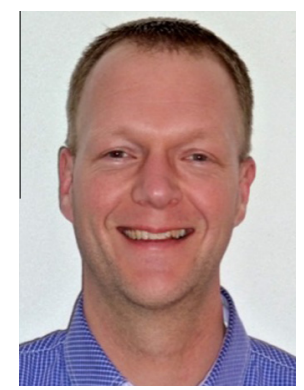

Marco Hoozemans works as an assistant professor at the Department of Human Movement Sciences at the Vrije Universiteit Amsterdam, The Netherlands, since 2001. His main research interests focus on the functioning and mechanical loading of the upper extremities in work and sports and on the functioning of the musculoskeletal system in relation to orthopaedic surgery (in older adults). He coordinates and teaches several courses on research methodology in musculoskeletal physiotherapy, epidemiology and (bio)statistics.

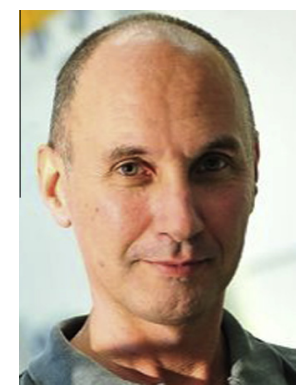

DirkJan Veeger is affiliated with the Department of Human Movement Sciences, Vrije Universiteit Amsterdam and the Faculty of Mechanical Engineering, section Biomedical Engineering, Delft University of Technology. After his M.Sc. in Human Movement Sciences (Amsterdam, 1984) and Ergonomics (London, 1985) he returned to Amsterdam, where he received his Ph.D. in 1992 on Biomechanics of Wheelchair Propulsion. In 2000 he was part time appointed in Delft. His main research interest lies in the field of musculoskeletal mechanics, and especially the upper extremity. Most profound research subject is the relationship between structure and function, which has been applied to the shoulder in wheelchair propulsion, the effect of surgical interventions such as tendon transfers on muscle function and ADL. His specialties are 3D kinematics and UE anatomy in relation the musculoskeletal modelling.

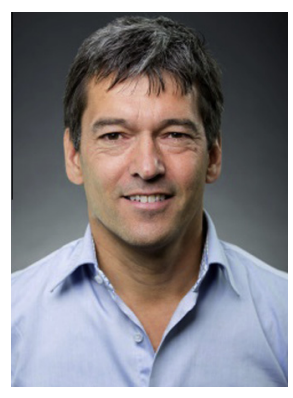

Michiel de Looze got his Ph.D. degree in Human Movement Sciences in 1992. Until 1998 he has worked as a researcher at the VU University in Amsterdam. The focus of his work was on biomechanics (torques and forces) and physiology (energy, fatigue) in occupational activities such as lifting, carrying, pushing and pulling. Since 1998 he is working at TNO (Netherlands Institute for Applied Research), where he is involved in research and innovation projects in close cooperation with industry. His research focus is on robot systems and their impact on human performance and wellbeing. It covers physical, cognitive and psycho-social aspects of the work load. His research gives input to the design and implementation of new technologies (wearable robots, co-bots, cognitive support systems), mainly in manufacturing and logistics. Since 2005 he has a (part-time) appointment at the VU University in Amsterdam as a Professor in Production Ergonomics. 